\title{
Gradiente colorimétrico de méis provenientes do pasto apícola da região de Ribeira do Pombal, Bahia
}

\author{
Dráuzio Correia Gama ${ }^{1}$, Favízia Freitas de Oliveira ${ }^{2}$, Genésio Tâmara Ribeiro ${ }^{3}$, Janisson Batista de Jesus ${ }^{4}$
}

RESUMO: Entende-se a coloração do mel como uma das características sensoriais que podem variar de acordo com a sua origem floral. O presente trabalho foi realizado com o objetivo de caracterização do gradiente colorimétrico de méis apícolas, conforme a flora do município de Ribeira do Pombal, Bahia. Para as análises, foram utilizadas 934 amostras de méis disponibilizados pela Cooperativa dos Apicultores de Ribeira do Pombal, Bahia (COOARP), referentes à safra regional do ano de 2015. As cores foram determinadas utilizando Colorímetro Analisador da Cor de Mel modelo Hanna HI 96785C, seguindo a escala Gama de Cor Pfund $(\mathrm{mm})$, conforme escala de cor adotada pelo USDA (Departamento de Agricultura dos Estados Unidos da América) e utilizando glicerina como padrão de aferimento. Para a comparação quanto ao tipo de pasto apícola no município de Ribeira do Pombal, utilizou-se de levantamento de plantas visitadas pela abelha Apis mellifera scutellata (Lepeletier, 1836) (Hymenoptera, Anthophora, Apidae) realizado por Almeida (2007), durante os 12 meses do ano de 2006. Dentre amostras de méis analisadas, 81,55\% correspondeu à cor Âmbar Claro. Embora a riqueza de espécies aponte tendência para méis claros, o fator abundância foi relevante e responsável por maior composição de méis escuros.

Palavras-chave: Característica sensorial, Apis mellífera scutellata, análises de méis.

\section{Colorimetric gradient of honey associated at flora from the region of Ribeira do Pombal-BA, Brazil}

ABSTRACT: The coloring of honey is understood as one of the sensorial characteristics that can vary according to its floral origin. The present work was carried out with the objective of characterizing the colorimetric gradient of bee honeys, according to the flora of the municipality of Ribeira do Pombal, Bahia. For the analyzes, were used 934 samples of honeys provided by the Beekeepers Cooperative of Ribeira do Pombal, Bahia (COOARP), referring to the regional harvest of the year 2015. The colors were determined using Honey Color Analyzer Model Hanna HI 96785C, following The Pfund Color Range scale (mm), according to the color scale adopted by the USDA (United States Department of Agriculture) and using glycerin as the benchmark. In order to compare the type of apicultural pasture in the municipality of Ribeira do Pombal, it was used a survey of plants visited by Apis mellifera scutellata (Lepeletier, 1836) (Hymenoptera, Anthophora, Apidae) carried out by Almeida (2007), during the 12 months of the year 2006. Among samples of honeys analyzed, 81.55\% corresponded to the Amber Light color. Although species richness points to trend for light honey, the abundance factor was relevant and responsible for higher composition of dark honeys.

Keywords: Sensorial characteristic, Apis mellifera scutellata, analysis of honeys.

\section{INTRODUÇÃO}

Obtido dos néctares das flores pelas abelhas, o mel pode ser classificado como monofloral, quando procede de flores de uma mesma espécie (possuindo características sensoriais, físico-químicas e microscópicas próprias), ou mel multifloral, quando procedente de diferentes origens florais. Em contrapartida ao mel floral, existe ainda o mel melato, que é formado, principalmente, a partir de secreções de partes vivas das plantas ou de excreções de insetos sugadores de plantas que se encontram sobre elas (honeydew) (BRASIL, 2000, CAMPOS et al., 2003). No Brasil, $70 \%$ da produção nacional são classificados como méis silvestres (EDNA et al., 2008), ou seja, méis multiflorais.

As abelhas africanas Apis mellifera scutellata Lepeletier (1836), originárias das áreas semiáridas sub-saarianas da África, foram introduzidas no Brasil em 1956, desencadeando acidentalmente o

\footnotetext{
Recebido em 24/05/2018; Aceito para publicação em 19/10/2018

${ }^{1}$ Universidade Estadual do Sudoeste da Bahia

${ }^{2}$ Universidade Federal da Bahia

${ }^{3}$ Universidade Federal de Sergipe

${ }^{4}$ Universidade Federal do Rio Grande do Sul

*E-mail: drauziogama@ hotmail.com
} 
processo de africanização, o qual deu origem às abelhas africanizadas criadas hoje na maior parte dos países da América do Sul (KERR, 1957, 1967) e responsáveis pela quase totalidade dos méis produzidos e comercializados nos Neotrópicos.

E quando se trabalha com mel, é comum encontrar variações na sua composição física e química, tendo em vista que variados fatores interferem na sua qualidade. Essa composição é influenciada pelas propriedades do néctar da espécie vegetal em que as abelhas visitam, considerando também o tipo da planta, clima, natureza do solo, estado fisiológico da colônia, estado de maturação do mel, condições meteorológicas e ambientais (CAMPOS, 1987, BRASIL, 2000, SILVA et al., 2004, MENDES et al., 2009).

De acordo com a instrução normativa que regulamenta a identidade e qualidade do mel, entende-se por características sensoriais a cor, sabor, aroma e consistência. A coloração do mel, por sua vez, varia de acordo com a sua origem floral, podendo ser quase incolor, âmbar, escuro e pardo escuro (BRASIL, 2000).

Aroucha et al. (2008), elucidam que a cor do mel está relacionada com a origem floral, podendo ser influenciada pelas condições climáticas durante o fluxo de néctar e a temperatura da colmeia. O tempo de estocagem, a luz, o calor e as possíveis reações enzimáticas podem ser apontados como outros fatores influenciando na coloração do mel.

No Brasil, em especial na região do semiárido nordestino, a vegetação nativa é responsável por méis apícola de variada coloração (ALMEIDA et al., 2016). Situação essa observada no município de Ribeira do Pombal, onde se tem o hábito de diferenciar dois tipos de pasto apícola ao longo do ano, denominados como "florada de inverno" (referente aos meses de chuva de maio e outubro) e a "florada de verão" (basicamente referindo-se às chuvas dos meses de novembro a abril) (ALMEIDA, 2007, GAMA et al., 2015a).

Considerando-se que as propriedades que conferem a cor do mel são influenciadas pelo tipo da vegetação e do clima de uma região, o presente trabalho foi realizado com o objetivo de caracterizar o gradiente colorimétrico de méis correlacionados à flora do município de Ribeira do Pombal, Bahia.

\section{MATERIAL E MÉTODOS Caracterização do local de estudo}

Localizado na região nordeste do estado da Bahia, entre as coordenadas UTM: X: 532863 e Y: 8832900; X: 572022 e Y: 8787285, Ribeira do Pombal possui extensão territorial de 762,212 $\mathrm{km}^{2}$ (BRASIL, 2010), fazendo parte da ecorregião do Raso da Catarina, caracterizada por solo arenoso, profundo e relevo plano, temperatura média de $24^{\circ} \mathrm{C}$.ano ${ }^{-1}$ e índice pluviométrico médio de 700 mm.ano ${ }^{-1}$ (VELLOSO et al., 2002).

O município de Ribeira do Pombal possui uma vegetação hipoxerófila predominantemente de porte arbóreo-arbustivo, arbustivo ralo a densa, pouco espinhosa, apresentando também áreas de pastagem e vegetação rala (GAMA et al., 2015b).

Abrigando uma população de 50.805 habitantes (BRASIL, 2013), com atividades econômicas baseadas em serviços, comércio e agropecuária, com especial destaque à apicultura (BRASIL, 2006). Ribeira do Pombal, com produção estimada em 450 toneladas, foi o município de maior produção de mel do país na safra de 2014, representando $14,3 \%$ da produção no Estado (BRASIL, 2015).

Nesse contexto, para a elaboração do presente trabalho, foram disponibilizados pela Cooperativa dos Apicultores de Ribeira do Pombal (COOARP) dados referentes à coloração de méis de 934 amostras (tipos) produzidos e comercializados pela entidade referente às safras da florada de inverno (maio a outubro) e verão (novembro a abril) do ano de 2015.

\section{Obtenção e processamento dos dados}

As amostras de méis disponibilizadas pela Cooperativa dos Apicultores de Ribeira do Pombal (COOARP), foram determinadas as cores com auxílio de Colorímetro Analisador da Cor de Mel modelo Hanna HI 96785C, seguindo a escala Gama de Cor Pfund(mm), relacionado ao padrão de cor adotado pelo USDA (Departamento de Agricultura dos Estados Unidos da América), utilizando glicerina como padrão. Nas análises foram determinadas as cores distribuídas em: Extra Claro (8-17mm); Branco (18-34mm); Âmbar Extra Claro (35-50mm); Âmbar Claro (51-85mm); Âmbar (86$114 \mathrm{~mm})$ e Âmbar Escuro (114mm - $\infty)$.

No que se refere ao tipo de pasto apícola responsável pelo gradiente colorimétrico dos méis relacionado, utilizou-se de resultados do 
levantamento das espécies vegetais visitadas pela Apis mellifera scutellata (LEPELETIER, 1836) em trabalho feito por Almeida (2007), durante os 12 meses do ano de 2006, realizado em área de remanescente de caatinga do município de Ribeira do Pombal, utilizando o método de trilhas em transecto percorrido na área testemunhando $\mathrm{e}$ registrando a visitações das abelhas Apis mellífera scutellata em espécies vegetais nectaríferas. Os dados foram processados em planilha Excel 2010.

\section{RESULTADOS E DISCUSSÃO}

Das 924 amostras de méis avaliadas, 756 (82\%) correspondem à cor Âmbar Claro (51-85mm); 101 (11\%) com a cor Âmbar Extra Claro (35$50 \mathrm{~mm}) ; 51(5,52 \%)$ com a cor Âmbar $(86-114 \mathrm{~mm})$; nove $(0,97 \%)$ com a cor Branco $(18-34 \mathrm{~mm})$ e sete $(0,76 \%)$ com a cor Âmbar Escuro $(114 \mathrm{~mm}-\infty)$. Na faixa de $8-17 \mathrm{~mm}$ (cor Extra Claro), não ocorreu nenhuma das amostras de méis avaliadas (Figura 1).

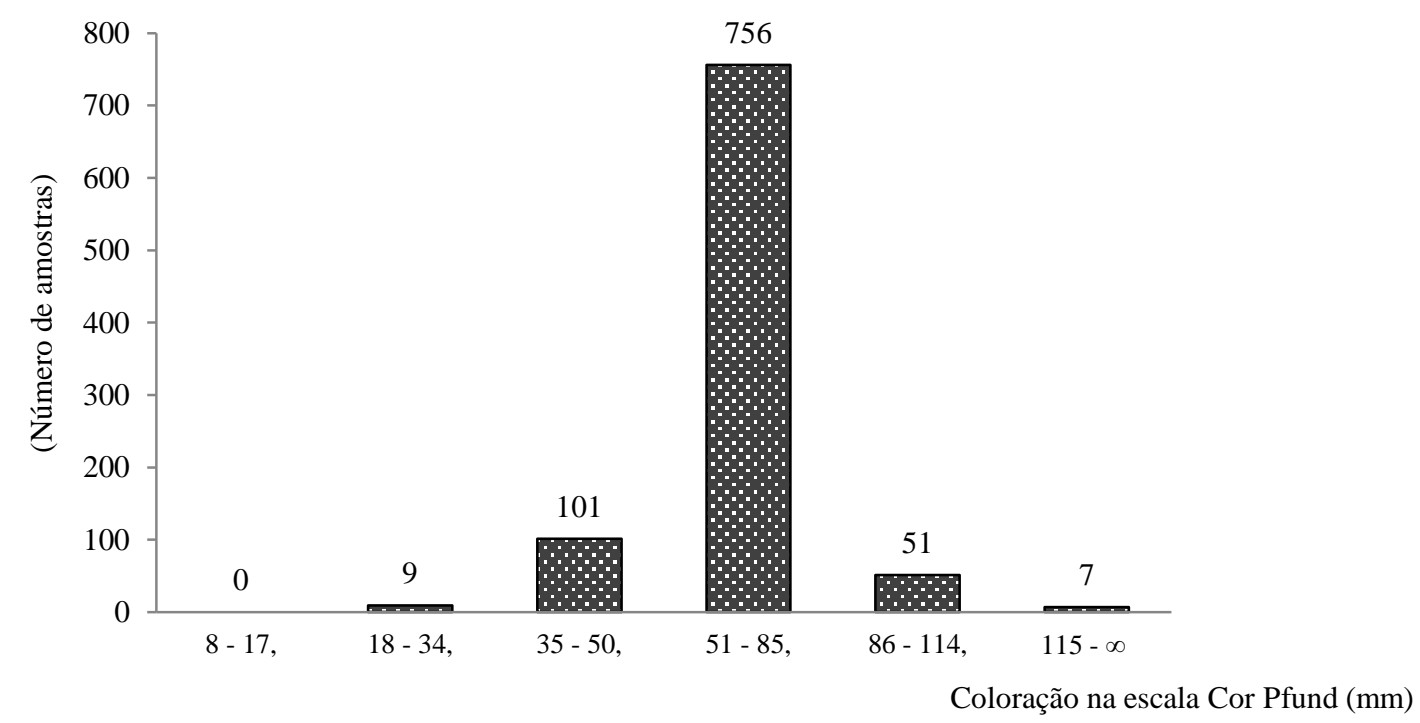

Figura 1 - Gradiente colorimétrica de méis apícolas classificados conforme Colorímetro Hanna mediante a escala padrão internacional Gama de Cor Pfund(mm), na região de Ribeira do Pombal, Bahia.

Moreti et al. (2006), em estudo analisando a cor de méis de seis Estados brasileiros (173 amostras da Bahia, 21 do Tocantins, 38 do Ceará, 52 do Piauí, 42 de Minas Gerais e 20 de Santa Catarina), observaram que do total de 346 amostras 44,52\% foi classificado na cor Âmbar-Claro e 17,05 \%, Âmbar Extra Claro, conforme a mesma escala Pfund do presente estudo.

Comparando a classe de cor Âmbar Claro, o encontrado em Ribeira do Pombal é superior ao de Moreti et al. (2006) com baixa diferença a cor Âmbar Extra Claro, levando em consideração que as amostras de méis de Moreti et al. (2006), são oriundos de pastos apícolas de climas e vegetação diversa dos Estados citados.

Todavia, a aproximação das taxas das duas escalas superiores de ambos os trabalhos, mostra-se ser uma possível caracterização comum para os méis no Brasil.
Por outro lado, como os méis em Ribeira do Pombal se apresentam na escala de cor acima de $51 \mathrm{~mm}(88,1 \%$ das amostras), são tidos, comercialmente, como méis escuros acarretando baixa valoração monetária e decisão na compra do produto, principalmente, quando se destina à exportação onde se priorizam os méis claros (abaixo de 30mm).

Em contra partida, a qualidade nutricional e medicinal dos méis escuros tem sido superior, conforme Almeida et al. (2016) onde asseguram que o mel escuro é indicativo de maior teor de fenóis e maior capacidade antioxidante. Onde Couto \& Couto (2002), afirma de maneira geral, que o mel escuro tem mais sais minerais do que o mel claro, podendo conter uma proporção de quatro a seis vezes mais sais minerais. Cortopassi-Laurino, Gelli (1991) também ressaltam que a cor mais escura é uma característica dos méis que contêm maiores quantidades de açúcares redutores. 
Com relação ao pasto apícola conhecido para o município de Ribeira do Pombal, conforme inventário realizado por Almeida (2007), considerando as espécies vegetais nectaríferas, foi identificado 46 espécies vegetais visitadas pelas abelhas Apis mellífera scutellata pertencente a 15 famílias botânicas, com destaque para Fabaceae, representada por $28,3 \%$ das amostras analisadas, seguida por Malvaceae (15,2\%), Euphorbiaceae $(10,9 \%)$, Verbenaceae $(8,7 \%)$ e Rubiaceae e Sapindaceae, ambas representadas por 6,5\%.

De forma similar, Gomes et al. (2009), em trabalho no município do Estado da Paraíba, identificaram 49 espécies de plantas pertencentes a 29 famílias botânicas preferencialmente visitadas pelas abelhas Apis mellifera scutellata, com destaque para as famílias Fabaceae (14,3\%), Anacardiaceae (12,2\%) e Euphorbiaceae (12,2\%).

Em áreas de vegetação nativa e de fruticultura irrigada em Petrolina (PE), Santos et al. (2006), entre janeiro de 2004 a maio de 2005, percorrendo as áreas escolhidas e observando o que eram visitadas por Apis melifera scutellata, registraram 51 espécies pertencentes a 25 famílias botânicas preferencialmente visitadas por essas abelhas com destaque para as famílias Fabaceae $(15,68 \%)$, seguida por Anacardiaceae, Convolvulaceae, Rubiaceae e Sterculiaceae ambas com $7,85 \%$ e por Lamiaceae e Euphorbiaceae com 5,88\% de representatividade cada.

Observações feitas em área de Caatinga na região de Itatim (BA), realizada entre setembro de 1996 e novembro de 1997, ao longo de um transecto, Aguiar et al. (2002) capturando com rede entomológica durante a visita às flores, registraram 33 espécies de plantas em 22 famílias botânicas onde Sterculiaceae (17\%), Erythroxylaceae (16\%), Fabaceae (14\%) e Rhamnaceae (9\%) foram as mais representativas das visitadas pelas abelhas Apis mellífera scutellata. Nesse caso, todavia, segundo os autores, poucas plantas foram importantes como fontes de pólen e néctar. E que vários estudos têm revelado um padrão comum de exploração dos recursos florais por A. mellifera, que visita um amplo espectro de plantas e concentra o forrageamento em poucas espécies (AGUIAR et al., 2002).

Em outro trabalho em diferente área da mesma região de Itatim (BA), Aguiar (2003), registrou as famílias Fabacaeae (17\%), Malvaceae (15\%), Sterculiaceae (14\%), Erythroxylaceae (9\%) e Rhamnaceae $(8 \%)$ como as mais visitadas pelas abelhas Apis mellifera scutellata o que permaneceu comum a presença marcante de famílias entre os dois trabalhos, somente alterando a ordem de número de espécies visitadas e a nova representatividade da família Malvaceae do último levantamento.

$\mathrm{Na}$ serra da Capivara (PI), Lorenzon et al. (2003), utilizando-se de redes entomológicas para a coleta das abelhas em visita às plantas, observaram que Apis mellífera scutellata forragearam em 32 espécies de 12 famílias botânicas, sendo Fabaceae maior representatividade com $37,5 \%$, seguida por Rubiaceae (15,6\%), Convovulaceae $(9,37 \%)$ e Acanthaceae, Euphorbiaceae e Laminaceae ambas com $6,25 \%$.

Trovão et al. (2009) em estudo no município de Caturité-PB, entre outubro de 2005 e julho de 2006, por intermédio de observações e monitoramentos das plantas em época reprodutiva, registraram 16 espécies de 9 famílias botânicas visitadas por Apis mellifera scutellata sendo as abelhas capturadas por redes entomológicas. E notaram maior frequência de visitação de Apis mellífera scutellata em plantas das famílias Fabaceae (50\%), seguida por Verbenaceae, Anacardiaceae e Caesalpiniaceae ambas em 12,5\% de ocorrência.

Nota-se assim que as famílias botânicas Fabaceae e Rubiaceae, presente no pasto apícola em Ribeira do Pombal, também são comuns a outros trabalhos em termos de representatividade.

Além dessas características, na consideração de pasto apícola de uma região, torna-se indispensável a distribuição dessas espécies nos seus diferentes estratos (arbóreo, arbustivo e herbáceo) e em diferentes épocas de floração ao longo do ano (florada de inverno e verão). Em que, considerando os dados levantados para o município de Ribeira do Pombal, observam-se afinidades do pasto apícola com o gradiente colorimétrico conforme se observa na Tabela 2. 
Tabela 2. Distribuição das espécies vegetais componentes do pasto apícola relacionadas com a cor do mel de acordo ao período de floração anual identificada na região de Ribeira do Pombal, Bahia.

\begin{tabular}{cccccc}
\hline \multirow{2}{*}{ Hábito } & \multirow{2}{*}{ Distribuição das espécies vegetais } & \multicolumn{2}{c}{ Cor do mel* } & \multicolumn{2}{c}{ Pasto apícola** } \\
\cline { 2 - 6 } & & Escuro (28,3\%) & Claro (71,7\%) & Florada de inverno & Florada de verão \\
\hline Arbustivo & $47,8 \%$ & $40,9 \%$ & $59,1 \%$ & $42,0 \%$ & $58,0 \%$ \\
Arbóreo & $17,4 \%$ & $25,5 \%$ & $75,0 \%$ & $25,0 \%$ & $75,0 \%$ \\
Herbáceo & $34,8 \%$ & $12,5 \%$ & $87,5 \%$ & $87,5 \%$ & $12,5 \%$ \\
\hline
\end{tabular}

Fonte: adaptado com base em Almeida (2007).

(*) considerando-se mel escuro acima de $51 \mathrm{~mm}$ e mel claro baixo de $51 \mathrm{~mm}$.

.(**) florada de inverno (maio a outubro); florada de verão (novembro a abril).

Conforme no inventário de Almeida (2007), as plantas visitadas pelas abelhas Apis mellifera scutellata por hábito, predomina como pasto apícola no município de Ribeira do Pombal espécies botânicas arbustivas, seguida por herbácea (Tabela 2), sendo que as espécies arbustivas são predominantemente visitadas na florada de verão e as herbáceas na de inverno.

E quanto ao estrato arbóreo, essa tem a menor representação de espécies visitadas $(17,4 \%)$ ocorrendo na florada de verão, com maior relevância na produção de méis claros $(75 \%)$, com destaque para duas espécies: Piptadenia moniliformes Benth (Fabaceae) (conhecida popularmente como quipé, angico de bezerro ou amargoso) e Gochnatia polymorfa Less. (Asteraceae) (conhecida popularmente na região como candeia).

Constata-se que, em todos os hábitos, predomina espécies que se pode produzir mel com coloração clara, onde o estrato herbáceo, embora representando a segunda menor riqueza em espécie (34,8\%), é o mais representativo em contribuição na produção de méis claros, com $87,5 \%$ da produção de mel prevalecendo-se na florada de inverno, com destaque nessa época para Althernanthera tenella Colla (Amaranthaceae), espécie herbácea conhecida por ervanço.

Em outros trabalho, a exemplo de Gomes et al. (2009), das espécies vegetais visitadas por Apis mellífera scutellata na região da Paraíba, 44,9\% corresponderam a espécies arbóreas. Santos et al. (2006), observaram Apis mellífera scutellata, em $41,17 \%$ de herbáceas, indicando a importância desse estrato na estação chuvosa (florada de inverno). Enquanto que as espécies arbóreas foram preferencialmente visitadas na florada de verão a exemplo das plantas Schinopsis brasiliensis Engl. (Fabaceae) e Myracrodruon urundeuva Allemão (Anacardeaceae).
Dessa forma, supõe-se que espécies vegetais do estrato herbáceo são comumente mais preferíveis pelas abelhas do gênero Apis sp.

Por outro lado, considerando ainda que as espécies do estrato herbáceo tenham grande contribuição para méis claros na florada de inverno, marcando essa importância significativa no município de Ribeira do Pombal, observa-se que ao longo do ano o gradiente correspondeu em mais de $80 \%$ em mel escuro (somando-se da florada de inverno e de verão), levando em consideração o gradiente acima de $51 \mathrm{~mm}$ de cor (Âmbar Claro), como se observa anteriormente na Figura 1.

Esse fato, provavelmente, tem relação pela grande contribuição da riqueza e, principalmente, da abundância de espécies arbustivas típicas da florada de verão, como exemplo a espécie Croton mucronifolius Muell (Euphorbiaceae), conhecida popularmente na região como catinga-de-cheiro, uma conhecida espécie geradora de mel escuro, confirmado pelos produtores. Somando a isso, devese levar em consideração que dentre as espécies herbáceas de florada de inverno, algumas são geradoras de mel escuro, como exemplo da espécie Hyptis multiflora Pohl. (Lamiaceae), conhecida popularmente na região como batônica.

Para Almeida et al. (2016), o mel está intimamente relacionada às espécies vegetais distribuídas em diferentes floradas típicas da região.

Nesse sentido, a variação colorimétrica com destaque para os méis escuros, ocorre em função da combinação de alguns fatores. Para estes, além de serem consideradas as características fisiológica e geneticamente intrínsecas de cada espécie, incluindo a tendência que cada planta tem em ofertar maior quantidade de néctar que outra, também deve ser levada em conta a disponibilidade do maior número de fragmentos contendo espécies do estrato arbustivo, contribuindo dessa forma para maior abundância de indivíduos das espécies desse estrato, 
a exemplo da já citada caatinga-de-cheiro, bastante abundante na região.

Ademais, o estrato herbáceo que, sendo típica de florada de inverno, sofre um comum agravo de torna-se indisponível, por estarem localizadas, predominantemente, em áreas de pasto ou em áreas agricultáveis, onde se torna um pasto apícola comprometido pelo cultivo ou pisoteio de gado.

Assim, pode-se diz que a Apis mellífera scutellata por ser uma espécie de forrageio caracteristicamente generalista, explorando toda e qualquer espécie vegetal nectarífera disponível, independente da época, supõem-se que elas acabam, por assim dizer, em privilegiar espécies arbustivas pela presença em riqueza e principalmente pela abundância das mesmas na área.

\section{CONCLUSÕES}

A caracterização colorimétrica do mel na região do município de Ribeira do Pombal, indica considerável concentração de méis na cor Âmbar Claro (51-85mm), representado por $81,55 \%$ das amostras estudadas.

Embora a riqueza de espécies aponte tendência para méis claros, o fator abundância provavelmente foi o responsável pela maior relevância na composição de méis em escuros, com $88,1 \%$ acima de $51 \mathrm{~mm}$, das amostras estudadas.

\section{AGRADECIMENTOS}

A Cooperativa dos Apicultores de Ribeira do Pombal (COOARP), pela disponibilidade dos dados para a realização do presente estudo.

\section{REFERÊNCIAS}

AGUIAR, C. M. L. Utilização de recursos florais por abelhas (Hymenoptera, Apoidea) em uma área de Caatinga (Itatim, Bahia, Brasil). Revista Brasileira de Zoologia, Curitiba, v. 20, n. 3, p. 457-467, 2003.

AGUIAR, C. M. L., MONTEIRO, V. M., SANTOS, G. M. M., RESENDE, J. J., FRANÇA, F., MELO, DE E. Plantas visitadas por apis mellifera L. (Hymenoptera, Apidae) em uma área de caatinga em Itatim, Bahia, Brasil. Sitientibus Série Ciências Biológicas, v.2, n. 1/2, p. 29-33, 2002.

ALMEIDA, A. M. M. DE., OLIVEIRA, M. B. S., COSTA, J. G., VALENTIM, I. B., GOULART, M. O. F. Antioxidant capacity, physicochemical and floral characterization of honeys from the Northeast of Brazil. Revista Virtual de Química, v. 8, n. 1, p. 57-77, 2016.

ALMEIDA, A. M. M. Flora visitada por Apis mellifera L. em Ribeira do Pombal, Bahia. 1. ed. Salvador: EBDA, 2007. 30p.

AROUCHA, E. M. M., OLIVEIRA, A. J. F. DE., NUNES, G. H. S., MARACAJÁ, P. B., SANTOS, M. C. A. Qualidade do mel de abelhas produzido pelos incubados da Iagram e comercializado no município de Mossoró/RN. Revista Caatinga, v. 21, n. 1, p. 211-217, 2008.

BRASIL, Instituto Brasileiro de Geografia e Estatísticas, Área Territorial Brasileira Consulta por Município, $2010 . \quad$ Disponível em: http://www.ibge.gov.br/home/geociencias/areaterritorial/a rea. Acesso: em fev. 2018.

BRASIL, Instituto Brasileiro de Geografia e Estatítica. Produção de mel por município na Bahia, 2015. Disponível em: http://www.ibge.gov.br/home/estatistica/economia/ppm/2 015/default_xls_perfil.shtm Acesso: em 10 mar. 2018.

BRASIL. Instituto Brasileiro de Geografia e Estatísticas. Censo Agropecuário, Cidades. 2006. Disponível em: <http://cidades.ibge.gov.br/xtras/temas.php?lang=\&codm un $=292660 \&$ idtema $=3 \&$ search $=$ bahia|ribeira-do-

pombal|censo-agropecuario>. Acesso em: 10 de jan. 2018.

BRASIL. Instituto Brasileiro de Geografia e Estatísticas. População Por Município, 2013. Disponível em: <http://cidades.ibge.gov.br/xtras/perfil.php?lang=\&codm un=292660\&search=bahia $>$. Acesso em: 10 de jan. 2018.

BRASIL. Ministério da Agricultura, Pecuária e Abastecimento. Defesa Animal. Legislações. Legislação

CAMPOS, G., DELlA-MODESTA, R. C., SILVA, T. J. P., BAPTISTA, K. E., GOMIDES, M. F., GODOY, R. L. Classificação do mel em floral ou mel de melato. Ciência e Tecnologia de Alimentos, v. 23, n. 1, p. 1-5, 2003.

CAMPOS, R. G. M. Contribuição para o estudo do mel, pólen, geleia real e própolis. Boletim Faculdade de Farmacia de Coimbra, v. 11, n. 2, p. 17-47, 1987.

COOARP - Cooperativa dos Apicultores de Ribeira do Pombal, Produção Relativo à Safra/2015, Anuário de Produção/COOARP, 2015.

CORTOPASSI-LAURINO, M., GELLI, D. S. Analyse pollinique, propriétes physico-chimiques et action antibactérienne dês miels d'abeilles africanisées Apis mellifera et de Méliponinés du Brésil. Apidologie, v. 22, n. 1, p. 61-73, 1991.

COUTO, R. H. N; COUTO, L. A. Apicultura: Manejo e produtos. 2. ed. Jaboticabal: FUNEP, 2002, 191 p. 
GAMA, D. C; NASCIMENTO JUNIOR, J. M. D; JESUS, J. B. de; FERNANDES, M. M. Utilização de SIG no estudo da distribuição de apiários no município de Ribeira do Pombal-BA. In.: CONGRESSO BAIANO DE APICULTURA E MELIPONICULTURA, 6. Anais..., p. 23 - 126, Ilheus, Bahia, 2015a.

GAMA, D. C; NASCIMENTO JUNIOR, J. M. DO; JESUS, J. B. DE; OLIVEIRA, D. G. de; FERNANDES, M. M. Tipologias vegetacionais de caatinga, município de Ribeira do Pombal-Ba. In.: SIMPÓSIO DE MUDANÇAS CLIMÁTICAS E DESERTIFICAÇÃO NO SEMIÁRIDO BRASILEIRO, 4. "Experiências e oportunidades para o desenvolvimento", Anais... Doc. 262 - Embrapa Semiárido, Petrolina-PE, 2015b.

GOMES, A. M. DE A., GUEDES, H. S., SOUZAJUNIOR, M. R. DE., GOIS, G. C., COELHO, M. DE S., EVANGELISTA-RODRIGUES, A. Flora apícola visitada por Apis mellifera (Hymeoptera, Apidae) no município de Salgado de São Félix, Paraíba. Magistra, v. 21, p. 62, especial, 2009.

KERR, W. E. Introdução de abelhas africanas no Brasil. Brasil Apícola, v. 3, p. 11-13, 1957.

KERR, W. E. The history of the introduction of African bees to Brazil. Africa Bee Journal, v.39, p.3-5, 1967.

LORENZON, M. C. A., MATRANGOLO, C. A. R., SCHOEREDER, J. H. A flora visitada pelas abelhas eussociais (Hymenoptera, Apidae) na Serra da Capivara, em caatinga do Sul do Piauí. Neotropical Entomology, v. 32, n. 1, p. 27-36, 2003.

MENDES, C. DE G., SILVA, J. B. DA., MESQUISTA, L. X. DE., MARACAJÁ, P. B. As análises de mel: revisão, Revista Caatinga, v. 22, n. 2, p. 07-14, 2009.
MORETI, A. C. DE. C. C., SODRÉ, G. DA S., MARChINI, L. C., CARVAlHO, C. A. L. DE. Cor de amostras de mel de Apis mellifera L. de diferentes Estados brasileiros. Indústria Animal, v. 63, n. 3, p. 159164, 2006.

por Assunto. Legislação de Produtos Apícolas e Derivados. Instrução Normativa n. 11, de 20 de outubro de 2000. Regulamento técnico de identidade e qualidade do mel. Disponível em: http://www.agricultura.gov.br/sda/dipoa/in_11_2000.htm. Acesso: 10 de fev. 2018.

SANTOS, R. F., KIILL, L. H. P;. ARAÚJO, J. L. P. Levantamento da flora melífera de interesse apícola no município de Petrolina-PE. Revista Caatinga, v. 19, n. 3, p. 221-227, 2006.

SILVA, C. L. DA., QUEIROZ, A. J. DA M., FIGUEIREDO, R. M. F. DE. Caracterização físicoquímica de méis produzidos no Estado do Piauí para diferentes floradas. Revista Brasileira de Engenharia Agrícola e Ambiental, v. 8, n. 2/3, p. 260-265, 2004.

TROVÃO, D. M. DE. B. M., SOUZA, B. C. DE., CARVALHO, E. C. D. DE., OLIVEIRA, P. T. B., FERREIRA, L. M. R. Espécies vegetais da caatinga associadas às comunidades de abelhas (Hymenoptera: Apoidea: Apiformis). Revista Caatinga, v. 22, n. 3, p. 136-143, 2009.

VELlOSO, A. L., SAMPAIO, E. V. S. B., PEREYN, F. G. C. Ecorregiões propostas para o Bioma Caatinga. Recife: APN/The Nature Conservancy do Brasil, 2002, $76 \mathrm{p}$. 